\title{
Comment je prescris de la témocilline ?
}

How to prescribe temocillin?

\section{Maxime Vallée ${ }^{1}$, Aurélien Dinh ${ }^{2}$}

${ }^{1}$ Service d'Urologie et de Transplantations rénales, CHRU de Poitiers, 2 Rue de la Milétrie, 86021 Poitiers, France

${ }^{2}$ Unité de maladies infectieuses, hôpital universitaire Raymond-Poincaré, UVSQ, AP-HP, 104, boulevard R.-Poincaré, 92380 Garches, France

Auteur correspondant : Maxime Vallée

maxime.vallee1786@gmail.com 


\section{Résumé :}

Les urologues sont de plus en plus souvent confrontés à des infections urinaires à bactéries multirésistantes et notamment les entérobactéries productrices d'une $\beta$-lactamase à spectre étendu (E-BLSE). La témocilline est une $\beta$-lactamine à spectre relativement étroit qui conserve une efficacité sur une majorité de ces entérobactéries productrice de BLSE/ou EBLSE et représente une alternative intéressante aux carbapénèmes pour le traitement des infections urinaires parenchymateuses documentées liées à ce type de bactéries. Nous fournissons ici un référentiel simple permettant une utilisation de cette molécule en pratique clinique courante.

Mots clés : Antibiotique, témocilline, infection urinaire

\section{Summary :}

Urologists are increasingly confronted with multi-drug resistant bacterial urinary tract infections, including enterobacteriaceae producing extended spectrum $\beta$-lactamase (ESBL). Temocillin is a $\beta$-lactam that retains efficacy on a majority of ESBL producing bacteria and represents an interesting alternative for the treatment of documented urinary tract infections associated with these bacteria. We provide here a simple reference allowing the use of this molecule in clinical practice.

Keywords : Antibiotic, temocillin, urinary tract infection 


\section{Introduction}

Devant l'augmentation majeure des résistances bactériennes et du nombre de décès liés à ces bactéries [1], l'urgence de trouver des alternatives efficaces aux traitements antibiotiques est aujourd'hui nécessaire tout comme optimiser le bon usage antibiotique [2]. En attendant ces alternatives, la prescription d'une antibiothérapie pour le traitement des infections urinaires devient de plus en plus complexe notamment lorsqu'il s'agit de traiter une infection liée à une entérobactérie productrice d'une $\beta$-lactamase à spectre étendu $(E-$ $B L S E)$. La témocilline, est une $\beta$-lactamine ancienne découverte dans les années quatrevingt. Son spectre étroit, considéré à l'époque comme un défaut, lui confère un un regain d'intérêt et ce d'autant plus qu'elle garde fréquemment une activité sur une grande variétés de $\beta$-lactamase et notamment les BLSE et les céphalosporinases hyperproduites [3].

L'objectif de cet article est de fournir un référentiel pratique permettant la prescription de cette molécule dans le cadre des infections urinaires nécessitant son recours.

\section{Indications}

Concernant son utilisation lors des infectiolons urinaires, les dernières recommandations SPILF-AFU mises à jour en 2018 ne recommandent son utilisation qu'en l'absence d'alternative de première ligne (fluoroquinolones, cotrimoxazole...) pour le traitement documenté des infections urinaires masculines ou des pyélonéphrites dues à des E-BLSE y compris en cas de bactériémies [4]. Ceci car il demeure actuellement des incertitudes concernant la dose et le seuil de CMI. Une étude française est en cours afin de lever certaines de ces incertitudes. Son utilisation n'est par ailleurs pas recommandée en probabiliste [4].

\section{Contre-indication}

La seule contre-indication est une allergie connue aux pénicillines.

\section{Spectre de la molécule et diffusion urinaire}

La témocilline a un spectre relativement étroit puisqu'elle n'est efficace que sur les entérobactéries à Gram négatif, ce qui exclut donc les bactéries à Gram positif, les anaérobies et Pseudomonas aeruginosa. Son intérêt réside principalement dans le fait qu'elle garde une efficacité contre une majorité des E-BLSE (60\% des souches sensibles pour une $\mathrm{CMI}$ retenue à $8 \mathrm{mg} / \mathrm{L}$ ) mais également contre des céphalosporinases hyperproduites $[3,4]$.

Les paramètres pharmacocinétiques et pharmacodynamiques sont encore mal connues mais semblent être similaires aux autres $\beta$-lactamines. La témocilline est essentiellement éliminée par voie urinaire sous forme active ce qui permet une diffusion importante dans les parenchymes des organes urinaires [3].

\section{Prescription}


La témocilline est une molécule à administration parentérale exclusive (IV ou IM). La posologie actuellement recommandée chez le patient normo-rénal est de $4 \mathrm{~g} / \mathrm{j}$ en deux injections ou $4 \mathrm{~g} / \mathrm{j}$ en IVSE après une dose de charge de $2 \mathrm{~g}[3,4]$. Certaines études plaident en faveur d'une administration de 6g/j (hors-AMM) lors d'infections graves ou lors d'infections nécessitant des posologies élevées du fait de la faible diffusion de la molécule dans certains tissus. C'est le cas de la prostate ou du testicule et de ses annexes où l'augmentation de la posologie semble préférable bien que non recommandée [3]. Cette augmentation de la posologie ne semble pas augmenter le risque d'effets indésirables.

Comme toutes $\beta$-lactamines (antibiotiques temps-dépendants) et notamment lors d'infection grave, son administration en continue est préférable afin d'obtenir des concentrations sanguines et tissulaires plus stables.

\section{Effets indésirables et surveillance}

Elle ne présente aucune particularité, ni effets indésirables autres que ceux des $\beta$ lactamines : réaction allergique ou troubles neurologiques avec convulsions essentiellement en cas de surdosages [3]. En cas d'insuffisance rénale avec un DFG inférieur à $60 \mathrm{~mL} / \mathrm{min}$, il faut adapter la posologie. La survenue de diarrhée ou de colite à Clostridium difficile a été décrite mais reste exceptionnelle.

\section{Quelles sont les différentes manières de prescrire la témocilline?}

Témocilline, dose de charge de $2 \mathrm{~g}$ par voie IV puis débuter immédiatement après $4 \mathrm{~g} / 24 \mathrm{~h}$ en IVSE.

$\mathrm{Ou}$

Témocilline, dose de charge de $2 \mathrm{~g}$ par voie IV puis débuter immédiatement après $6 \mathrm{~g} / 24 \mathrm{~h}$ en IVSE (hors-AMM).

Ou

Témocilline, $2 \mathrm{~g}$ par voie IV toutes les $12 \mathrm{~h}$.

$\mathrm{Ou}$

Témocilline, $3 g$ par voie IV toutes les $12 \mathrm{~h}$ (hors-AMM).

$\mathrm{Ou}$

Témocilline, $2 \mathrm{~g}$ par voie IV toutes les $8 \mathrm{~h}$ (hors-AMM).

Ou

Témocilline, $2 \mathrm{~g}$ par voie IM toutes les $12 \mathrm{~h}$ après reconstitution du produit avec $3,5 \mathrm{ml}$ de lidocaïne à $10 \mathrm{mg} / \mathrm{ml}$ (pour éviter les douleurs au point d'infection).

Ou

Témocilline, $2 \mathrm{~g}$ par voie IM toutes les $8 \mathrm{~h}$ après reconstitution du produit avec $3,5 \mathrm{ml}$ de lidocaïne à $10 \mathrm{mg} / \mathrm{ml}$ (hors-AMM).

NB : Molécule stable après reconstitution avec le solvant (eau pour injection, sérum physiologique ou glucose $5 \%$ ) pendant 24 heures ce qui permet la préparation d'une seule dose par jour lors d'administration en continue.

La témocilline est à prescription hospitalière et est rétroscédable par l'hôpital ce qui permet son utilisation avec des prestataires de soins en ambulatoire. 


\section{Conclusion}

La témocilline est une molécule qui représente aujourd'hui une alternative intéressante aux carbapénèmes pour le traitement des infections urinaires à E-BLSE et dont l'utilisation va être croissante. Elle ne doit être utilisée qu'en cas d'infection documentée à bactéries sensibles et à forte dose. Par ailleurs son coût pourrait également être un frein puisqu'une journée de traitement à une posologie de $4 \mathrm{~g} / \mathrm{J}$ revient environ à $120 €$.

\section{Conflits d'intérêt}

Les autres auteurs ne déclarent aucun conflit d'intérêts. 
Références

1. Cassini A, Högberg LD, Plachouras D, Quattrocchi A, Hoxha A, Simonsen GS, et al. Attributable deaths and disability-adjusted life-years caused by infections with antibiotic-resistant bacteria in the EU and the European Economic Area in 2015: a population-level modelling analysis. The Lancet Infectious Diseases. 2019;19(1);56-66.

2. Vallée $M$, Bruyère $F$. L'infectiologie urinaire, un métier d'avenir ? Progrès en Urologie. juin 2018;28(8回9):405回6.

3. Vallée $M$, Bruyère $F$, Roblot $F$, Brureau L. Temocillin and urinary tract infections. Prog Urol. 2017;27(12):609-17

4. Caron F, Galperine $T$, Flateau $C$, Azria R, Bonacorsi S, Bruyère F, et al. Practice guidelines for the management of adult community-acquired urinary tract infections. Médecine et Maladies Infectieuses. août 2018;48(5):327?58. 